\title{
Influence of opposite elevation on the occupancy level of the tracks of sorting park
}

\author{
Sergei Bessonenko, ${ }^{1,}$, Konstantin Kornienko ${ }^{1}$, Iuliia Tanaino $^{1}$ \\ ${ }^{1}$ Siberian Transport University, Dusi Kovalchuk st., 191, 630049, Novosibirsk, Russia
}

\begin{abstract}
The purpose of this paper is to study the effect of the location and magnitude of opposite elevation on the occupancy level of the tracks of sorting park. The subject of the study is the effect of the magnitude and location of opposite elevation on the sorting tracks occupancy level. The object is the tracks of sorting park. For the study, a simulation model of the traffic of the cut in sorting park was used, taking into account the reverse movement, "SortPark". On the basis of this model, it was concluded that when the target point is moved farther from the braking position, the distance traveled by the cut first increases, and then decreases. This is due to the fact that when stopping the cut on the opposite elevation, it can start to move backward. On the basis of this, it was concluded that the useful length of the sorting yard track for the first detaching limited by the point of change of gradient from grade to opposite elevation. In view of this conclusion, the formula for determining the sorting track occupancy level was improved.
\end{abstract}

\section{Introduction}

Today, the railway is one of the main elements of the transport network of the Russian Federation and the whole Europe. Therefore, special attention is paid to the development of the railway. One of the directions of the development of railway transport is the modernization of sorting stations [1]. The development of sorting stations is impossible without the improvement of hump yards.

\section{Actuality}

One of the elements of the sorting complex is the sorting park. It accumulates a car traffic volume for the further formation of trains. In the process of accumulation, collisions of cars with exceeding of the permissible speed are possible due to the non-conformity of the profile to design decisions, and also to irrational design solutions [2]. As a result of the collision, damage to cars and commercial defects associated with cargo shifts or stake fracture can occur. The occurrence of such defects leads to an increase in the time of accumulation of cars, as well as the time for the delivery of cargo. Acceleration of cargo delivery is impossible without reducing the residence time of cars under the accumulation

\footnotetext{
* Corresponding author: kkonstantini@mail.ru
} 
[3].

Many scientists have been addressed the issue of the profile of the downhill part of the hump yard [4-6]. At the same time, in the "Rules and standards for design of sorting devices on railway tracks of $1520 \mathrm{~mm}$ " [7], the sorting park is considered only up to the nominal design point. Therefore, the issues related to the design of the sorting park are still insufficiently studied. At the same time, a change in the profile of a sorting park is one of the main problems in sorting complexes [8-9]. In this regard, more and more research is now devoted to the sorting park [10-13].

In [14], V.N. Sokolov highlights the high processing capacity as one of the most important parameters of the hump yards typical for the Russian Federation. Because the sorting tracks in Russia accumulate on average in 4-5 times more cars per day than in the countries of Western Europe and the United States. Consequently, in foreign sorting complexes, there is no need to increase the speed of detaching of cuts and the speed at which cars are delivered to the park. Because of this, the problem of ensuring the safety of collisions of cuts in the sorting park and the minimization of "windows" is not so acute.

The influence of the external environment and the design of hump devices on the sorting track occupancy level were considered by S.V. Karasev [15-16]. In his work, he concludes that the high level of sorting track occupancy should be determined by two factors: minimization of "windows" and collision with permissible speed. The model developed by him [17] takes into account the processed car traffic volume and cut traffic volume, and also takes into account the total specific resistance to car traffic on the whole general set of possible values. The main conclusions were made about the influence of the height of the hump yard and the weather conditions on the volume of the shunting work and on the amount of car backing outs. At the same time, the effect of the sorting track profile on the level of its occupancy was not considered.

In this paper, two conditions will be understood under the sorting track occupancy level: the minimization of "windows" and the collision of cuts with an allowable speed.

In addition to the longitudinal profile, the park braking position also has a great influence on the sorting track occupancy level. In study [18], the author identifies a method for reducing the exit speed of a cut into the park using beam retarders as the most effective method. In case if the accuracy of exit speed is not sufficient, the use of point retarders with beam ones is recommended. The equipment of the sorting park with additional devices is one of the most effective ways to improve the level of track occupancy $[9,15,19]$. But this method requires large capital costs and is complicated in technical implementation and maintenance. The conclusion was made in paper [20] that the incorrect operation of the retarder leads to deterioration in the level of track occupancy.

Based on the above, it can be concluded that many factors affecting the sorting track occupancy level have been investigated at the current time, but the issues of the influence of the track profile and other conditions on the its occupancy level have not been fully studied.

The actual profile of the sorting park can be distorted by many factors [8]. Changing the profile leads to the fact that occupancy level of tracks also changes. One of the important factors is the formation of opposite elevations within the useful length of the sorting tracks.

\section{Object and purpose}

The purpose of this paper is to study the effect of the location and magnitude of the opposite elevation on the occupancy level of sorting park tracks.

The object of the study is the tracks of sorting park.

The subject of the study is the effect of the magnitude and of the opposite elevation on 
the occupancy level of tracks.

To achieve this goal, it is necessary to solve the following tasks:

1. To assess the influence of the profile of the sorting park on the position of the target point when releasing the cut to the free track.

2. Derive the formula for determining the level of occupancy of free track taking into account the position of the target point.

\section{Methods of research and subject of research}

The developed simulation model was used for the study [12, 13, 21]. In this paper, an example of the study of a single profile of the real track of a sorting park is given. The track is considered from the end of the park retarder to the insulating joints in the yard neck. The values of the slopes and their length are shown in Figure 1.

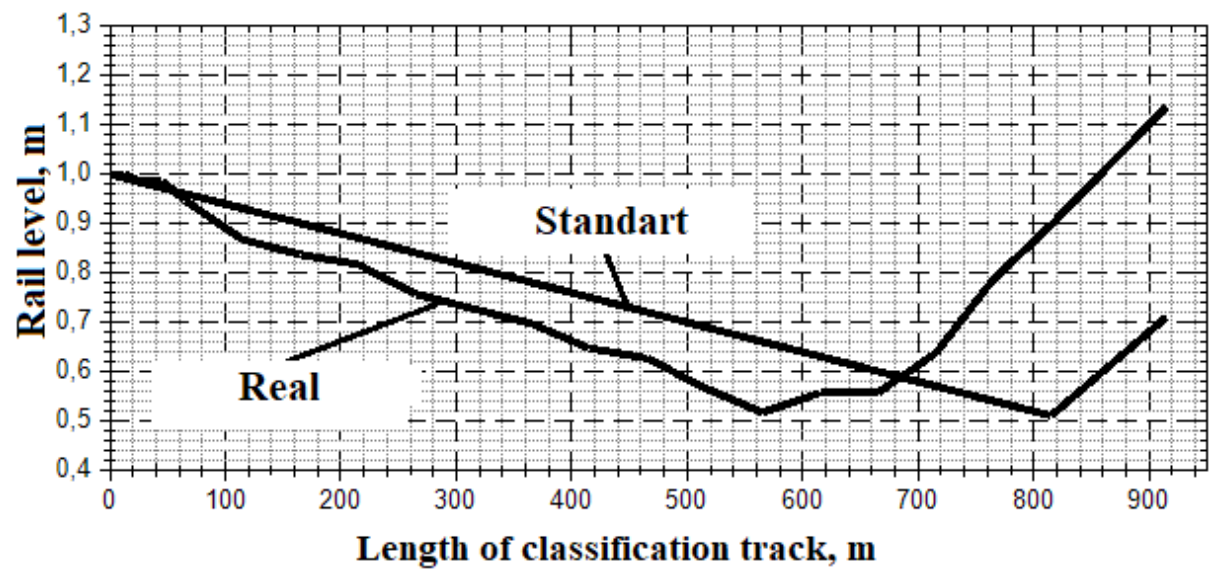

Fig. 1. Profile of the marshaling yard track.

\section{Calculation of the optimal target point}

In $[22,23]$ it is stated that the cut, which is let out on the free track, must reach the end of the sorting park, i.e. up to the brake shoe holders in the yard neck. This means that the cut should stop at the end of the sorting park on the opposite elevation. In this case, after stopping on the opposite elevation, the cut can start to move backward under the action of gravity. In the work [13], the author concludes that the position of the point of complete stop must satisfy two conditions:

- The speed of the cut at this point is equal to zero.

- The starting condition, including in the opposite direction, is not fulfilled for the cut.

On the basis of this, it can be concluded that the points of a full stop will be very different from the stopping points without taking into account the reverse movement (the movement of the cars back towards the hump of the yard under the action of gravity). To study this difference, a simulation modeling of the movement of 10,000 single-car cuts with different basic specific resistance and different exit speed was carried out. For the experiment, the climatic conditions were assumed to be the same for all runners. After modeling, the probabilities of stopping the cut at different points of the sorting park were derived. On the basis of these probabilities, the distribution density curves of the stopping points of the cuts were constructed with and without the reverse movement taken into account. This graph is shown in Figure 2. 


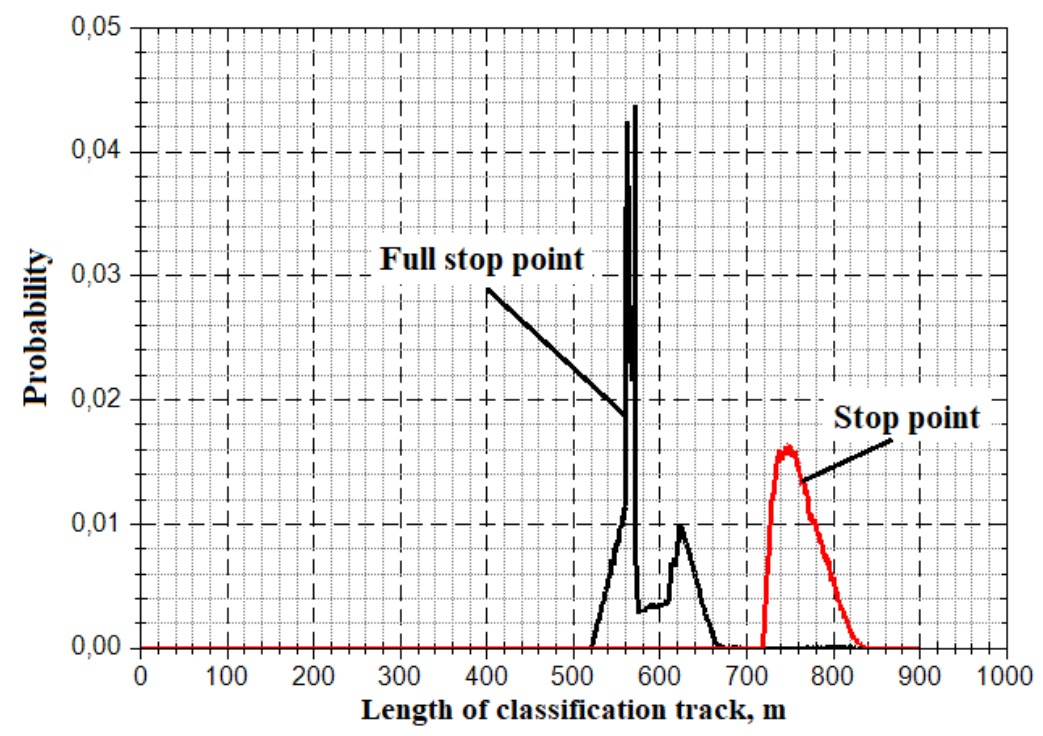

Fig. 2. The distribution density curves of the stopping points of the cuts with and without the reverse movement taken into account.

Analyzing Figure 2, we can conclude that the points of the full stop of the cut are displaced closer to the middle of the track. This is due to the fact that the opposite elevation on the track starts with a mark of 573 meters and not with 823 as it should be according to the rules [7]. In this regard, most of the cuts sent on this track will start to move back after a stop. Figure 3 shows an example of the dependence of the position of the full stop point of the cut on the position of the target point for one cut.

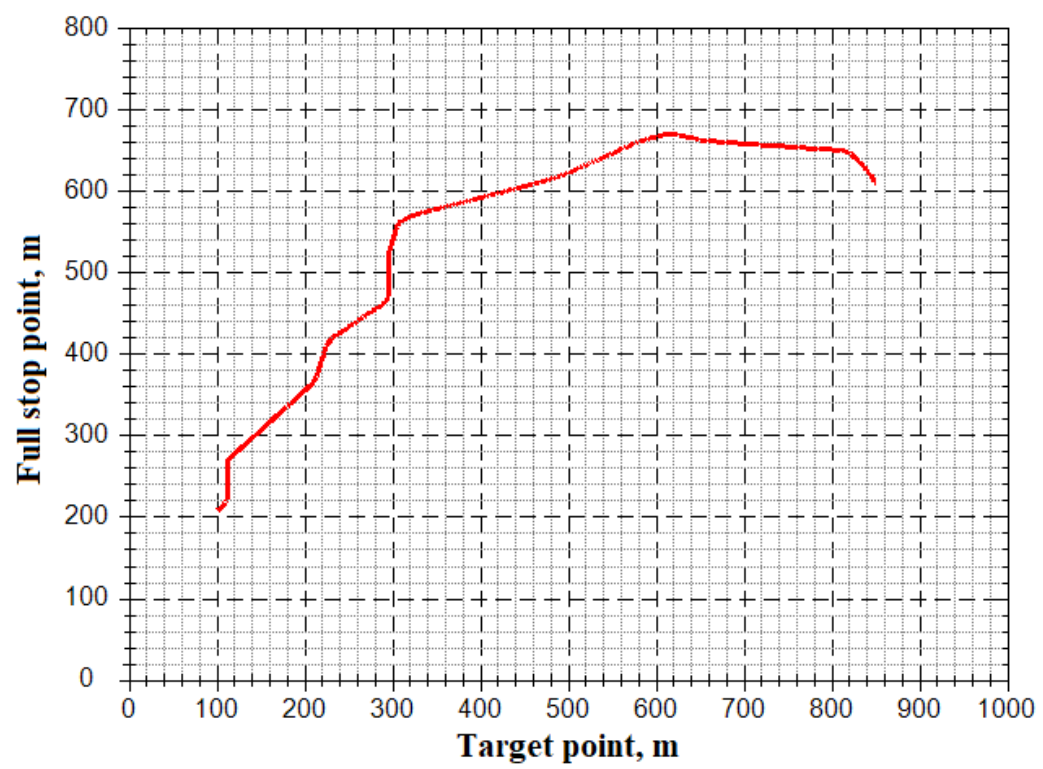

Fig. 3. The dependence of the position of the full stop point of the cut on the position of the target point.

On the basis of Figure 3, we can conclude that when the position of the target point is 
shifted up to 620 meters, the run of the cuts also increases. In this case, meters there is a change of gradient after 570, and when the cut stops at the opposite elevation, it will move back towards the hump of the yard. The probability of rolling down the cut will depend on the running characteristics of the car and on the natural conditions. For this cut, the target point should be set at 620 meters, since the traveled path will be maximum at this point.

If we analyze Figure 2 and Figure 1, then we can conclude that the optimal target point for the free track is the point of a change of gradient of the sorting track. Therefore, this is the point where the grade changes to an opposite elevation.

Based on the above, it can be concluded that the profile of the sorting track will have a huge impact on the target point. In this case, the target point should be assigned at a point of 670 meters, and this point lies at the distance of 150 meters from the design target point (opposite elevation should start at a point of 820 meters) [7].

\section{Index of sorting track occupancy level}

Criterion of sorting track occupancy level is the index of sorting track occupancy level [22]:

$$
K_{\text {OCCUP }}^{i}=\frac{N_{C A R}^{i} * L_{C A R}}{L_{\text {FREE }}^{i}-L_{\text {ISTOL }}^{i}}
$$

where $N_{C A R}^{i}$ - the number of cars entering the i-th track in this detaching, pcs;

$L_{C A R}$ - the length of conventional car, $14,5 \mathrm{~m}$;

$L_{F R E E}^{i}-$ the distance of run of the first cut during the detaching on the i-th track, m;

$L_{I S T O L}^{i}$ - the length of free area of i-th track after the detaching, $\mathrm{m}$.

Formula (1) is used to determine the sorting track occupancy level with one detaching. If we consider the entire process of accumulation of cars on the track from the release of the first cut to the first backing out, formula 1 can be written in the following form:

$$
K_{\text {OCCUP }}^{i}=\frac{N_{C A R}^{i} * L_{C A R}}{L_{\text {TRACK }}^{i}}
$$

where $L_{T R A C K}^{i}$ - the length of the i-th track of the sorting park from the end of the park brake position to the brake shoe holders in the yard neck, $\mathrm{m}$.

The index of sorting track occupancy level is set in departmental instructions and must be no less than 0.8 [22]. Accordingly, the following number of cars should be placed on a sorting track of 923 meters long:

$$
N_{C A R}^{i}=\frac{0,8 * 923}{14,5}=50,9 \approx 50(p c s)
$$

In case if an opposite elevation starts from a mark of 573 meters, then the useful track length should be considered as the distance from the park brake position to the beginning of the opposite elevation. Accordingly, in this case, even if in the interval from 0 to 573 meters we have the maximum occupancy of tracks 1 (i.e. the cars will collide with each other at an acceptable speed and no "windows" will be formed), then the track can fit at all: 


$$
N_{C A R}^{i}=\frac{1 * 573}{14,5}=39,5 \approx 39(p c s)
$$

Based on this, it is possible to calculate the occupancy level of the whole track:

$$
K_{\text {OCCUP }}^{i}=\frac{39,5 * 14,5}{923}=0,62
$$

Accordingly, the conclusion can be made that due to the fact that the opposite elevation starts at the mark of 573 meters, the index of sorting track occupancy level is less than the allowable by 0.18 . When calculating the number of cars, it was accepted that all cars will reach the previous cuts and collide with the permissible speed. Taking this into account, we can conclude that the index differs from the set one by 0.38 . This means that this track will fit 11 wagons less before backing out than is required by the standard.

Formula (2) can be used to calculate the sorting track occupancy level in case if the target point is the brake shoe holders at the end of the track. If the target point is the point of change of gradient, the formula for calculating the index of sorting track occupancy level for the free track will be:

$$
K_{\text {OCCUP }}^{*}=\frac{K_{O C C U P}^{N O M} * L_{T A R . P,}}{L_{T R A C K}}
$$

where $K_{O C C U P}^{N O M}-$ nominal index of sorting track occupancy level. The index established by the automated detaching system for a given track;

$L_{\text {TAR.P, }}$ - the length from the park braking position to the target point, $\mathrm{m}$.

Table 1 shows the results of the study of the index of sorting track occupancy level depending on various positions of the target point.

\begin{tabular}{|c|c|c|c|c|c|}
\hline$L_{\text {TAR.P }}$ & $K_{\text {OCCUP }}^{\text {NOM }}$ & $\mathrm{N}$ & $K_{\text {OCCUP }}^{*}$ & $\mathrm{~N}^{*}$ & $\Delta \mathrm{N}$ \\
\hline \multirow{5}{*}{600} & 0,8 & 51 & 0,52 & 33 & 18 \\
\hline & 0,85 & 54 & 0,55 & 35 & 19 \\
\hline & 0,9 & 57 & 0,59 & 37 & 20 \\
\hline & 0,95 & 60 & 0,62 & 39 & 21 \\
\hline & 1 & 64 & 0,65 & 41 & 22 \\
\hline \multirow{3}{*}{700} & 0,8 & 51 & 0,61 & 39 & 12 \\
\hline & 0,9 & 57 & 0,68 & 43 & 14 \\
\hline & 1 & 64 & 0,76 & 48 & 15 \\
\hline \multirow{3}{*}{800} & 0,8 & 51 & 0,69 & 44 & 7 \\
\hline & 0,9 & 57 & 0,78 & 50 & 8 \\
\hline & 1 & 64 & 0,87 & 55 & 8 \\
\hline \multirow{3}{*}{850} & 0,8 & 51 & 0,74 & 47 & 4 \\
\hline & 0,9 & 57 & $\mathbf{0 , 8 3}$ & 53 & 5 \\
\hline & 1 & 64 & 0,92 & 59 & 5 \\
\hline
\end{tabular}

Table 1. Results of the study of the dependence of the actual index of sorting track occupancy level on the position of the target point and the nominal index.

Note:

$\mathrm{N}$ - nominal number of cars, pcs. In other words, the number of cars that can fit in this 
track if the nominal occupancy index is met;

$\mathrm{N}^{*}$ - actual number of cars, pcs. In other words, the number of cars that entered this track under the condition that the position of the target point is shifted;

$\Delta \mathrm{N}$ - the difference between the nominal and the actual number of cars.

According to Table 1, the following conclusions can be drawn:

The target point has a very strong effect on the index of sorting track occupancy level;

The further the target point is located, the greater the index of sorting track occupancy level;

Because the optimal index of sorting track occupancy level is the index 0.9, then when the opposite elevation profile of the sorting park is leveled to the normative value, the actual index of sorting track occupancy level will be 0.83 . Therefore, the track will fit in 13 cars more than at the moment.

To verify the correctness of the hypothesis, a simulation modeling of the occupation process of the sorting park track was carried out using the "SortPark 2" program [24]. The distribution of the probability of the sorting track occupancy level at the first detaching is shown in Figure 4:

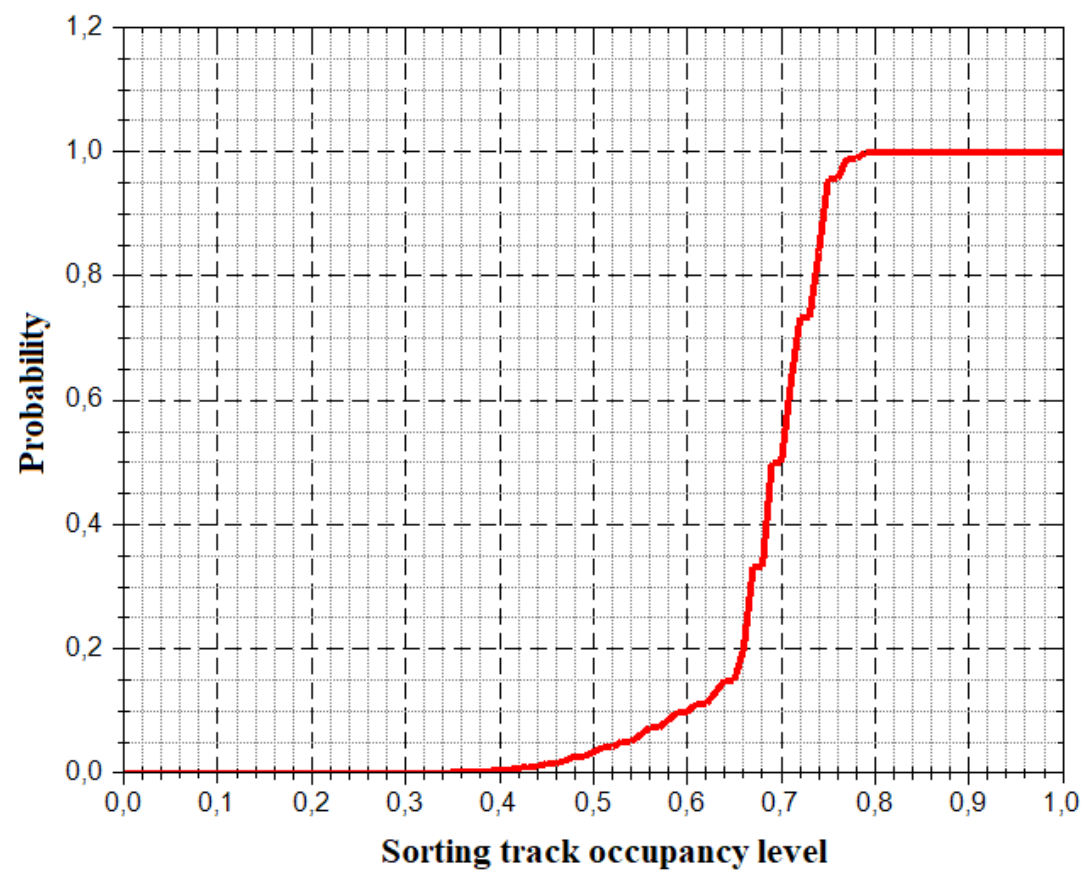

Fig. 4. The distribution of the probability of the sorting track occupancy level at the first detaching.

On the basis of Figure 4, the following conclusions can be drawn:

- The average index of sorting track occupancy level in the simulation modeling is 0.7. The index obtained by formula (3) is less by 0.1 . This is due to the fact that the point 573 meters was taken as the target point (after which an opposite elevation and a flat section follow) when calculating by the formula (3). Because of this, a large number of cuts stopped on this opposite elevation will not start to move backwards. If we take the target point at 673 meters, then the indexes obtained by formula (3) and by simulation modeling of the track occupation will be equal.

- The conducted study of other tracks shows that the index obtained by the formula (3) in 
most cases coincides with the average value of the index of occupancy level obtained in simulation modeling. The use of formula (3) makes it possible to calculate (without using significant software and time tools) how will increase the sorting track occupancy level at the first detaching, when the profile is leveled.

\section{Conclusions}

Based on the study, the following conclusions can be drawn:

1. Analysis of the real profiles of the sorting station shows that the actual index of sorting track occupancy is much lower than the permissible on many tracks. This leads to a decrease in the time between breaking outs, and, consequently, to an increase in the technological interval of the hump yard;

2. When releasing a cut to the free track, the target point should be determined taking into account the profile of the sorting park. If there is a large opposite elevation in the sorting park, the target point should be assigned the point of change of the gradient from the grade to the opposite elevation. In this case, the target point influences the sorting track occupancy level.

3. The formula of the actual index of sorting track occupancy level for the free track is corrected, taking into account the target point, which allows calculating the sorting track occupancy level more accurately.

4. The corrected formula was verified using a simulation model of the track occupation process. The average value of the index of sorting track occupancy level of the simulation model coincides with the value calculated by the formula.

5. It is revealed that the index of sorting track occupancy level is below the permissible value on tracks with a long opposite elevation. In this case, profile leveling will increase the index.

\section{Acknowledgements}

The authors are grateful to the departments "Management of operational work" and "Railway stations and nodes" of the Siberian State Transport University and employees of the Insk distance of signaling, centralization and blocking for assistance in writing this paper. This study was carried out with the support of the Government of the Novosibirsk Region.

\section{References}

1. http://doc.rzd.ru/doc/public/ru?STRUCTURE_ID=704\&layer_id=5104\&id=6396

2. K.I. Kornienko, Vestnik of the Siberian State Transport University, 1 (2017)

3. O.P. Yugrina, Yu.A. Chechulina, L.S. Kazantseva, Chronicles of the united fund of electronic resources science and education 1, 29 (2016)

4. V.A. Kobzev, Science and technology of transport, 17-20 (2014)

5. A.N. Shabelnikov, A.V. Savrukhin, V.A. Kobzev, V.N. Sokolov, ASI 7, 9-12 (2016)

6. D.V. Osipov, A.A. Klimov, Transport of the Urals 4(47), 63-68 (2015)

7. Rules and regulations for the design of sorting devices on railway tracks of $1520 \mathrm{~mm}$ (Tehinform, Moscow, 2003)

8. A.N. Shabelnikov, Automation, communication, informatics 1, 6-8 (2013) 
9. A.N. Shabelnikov, Railway transport 8, 54-55 (2010)

10. K.I. Kornienko, Transport infrastructure of the Siberian region, 80-83 (2017)

11. T.I. Starostina, Analysis of the structural features of the longitudinal profile of the sorting tracks of the existing sorting stations, Innovative technologies of the new millennium: collection of papers of the International Scientific and Practical Conference. Part 2 (AERTERNA, Ufa, 2016)

12. E.A. Akhmaev, S.A. Bessonenko, V.V. Borisov, K.I. Kornienko, Transport of the Urals 4(55), 49-53 (2017)

13. K.I. Kornienko, Transport: science, technology, management 11, 36-40 (2017)

14. V.N. Sokolov, Automation, communication, informatics 2, 8-9 (2011)

15. S.V. Karasev, Influence of hump yard design, car traffic volume structure and external environment on the sorting tracks occupancy level, The thesis for the degree of $\mathrm{PhD}$ of Technical Sciences: 05.22.08 (Novosibirsk, 2003)

16. V.I. Zhukov, S.V. Karasev, O.V. Kutsenko, Simulation of the occupation process of a track of accumulation of cars in a sorting park, Improving the operational work of railways: Collection of scientific papers (Novosibirsk, 2002)

17. S.V. Karasev, Study of the joint influence of the height of the hump yard, the structure of train and wind conditions on the occupation of the track of accumulation of cars in a sorting park, Materials of scientific and technical conference "Science and youth of the XXI century" (Novosibirsk, 2002)

18. A.A. Nazarov, Science and progress of transport 4(64), 47-54 (2016)

19. S. Zarecky, Transport Problems, Tom 3 4(1), 87-95 (2008)

20. D.N. Kozachenko, S.V. Grevtsov, T.V. Bolvanovskaya, Science and progress of transport 4(64), 37-46 (2016)

21. K.I. Kornienko, Program for simulation modeling of the speed of cut operation in the sorting park "SortPark" Certificate of registration of the electronic resource United Foundation of Electronic Resources “Science and Education” № 22848 of 06/06/2017

22. K.I. Kornienko, Chronicles of the United Foundation of Electronic Resources Science and Education 6(97), 30 (2017)

23. A.N. Shabelnikov, Automation, communication, informatics 3, 9-11 (2009)

24. I.A. Olgeizer, Automation, communication, informatics 11, 27-28 (2007) 